\title{
Goal-oriented error estimation for fluid-structure interaction problems
}

\author{
Th. Richter $^{\dagger}$
}

July 6, 2011

\begin{abstract}
In this work, we present an adaptive finite element method for the numerical simulation of fluid-structure interaction problems. The coupled system is formulated in a variational monolithic Arbitrary Lagrangian Eulerian framework. We derive methods for goal-oriented error estimation and mesh adaptation with the dual weighted residual method. Key to this error estimator is a Petrov-Galerkin approach for deriving the variational formulation of the coupled system. The developed method is applied to two and three dimensional stationary benchmark problems to demonstrate its efficiency.
\end{abstract}

\section{Introduction}

Fluid-Structure interaction is part of various technical problems: the flow of blood in the heart is substantially influenced by the movement of the heart and the vessels as well as the heart valves. In aerodynamics, the elastic behavior of the wing demands for careful consideration for a proper simulation. In ship design, the interaction of the propeller with the water needs to be studied.

All these problems are naturally three dimensional. The computational effort for such a simulation is immense. The use of locally refined meshes is an effective remedy in the case of large three dimensional problems. While adaptive finite element methods have a long tradition and are well established for flow [OWA93] and structural [AO97, Ver96] problems, the consideration of multi-physics problems is new.

In most technical applications one is not interested in global error norms but in certain functional outputs of the solution like drag- or lift-coefficients in aerodynamics or like bending-moments in structure dynamics. We aim at estimating the error and optimizing the discretization with regard to these error functionals. First work on a posteriori error estimation with help of a duality technique has been done by Eriksson, Estep, Hansbo and Johnson [EEHJ95]. This method has been extended to the Dual Weighted Residual

${ }^{\dagger}$ University of Heidelberg, Institute for Applied Mathematics, INF 293, 69120 Heidelberg, Germany,

thomas.richter@iwr.uni-heidelberg.de 
Method by Becker and Rannacher (DWR-method) [BR01] for goal-oriented error estimation and automatic mesh adaption. With the dual weighted residual method, a general framework for estimating errors in output functionals is given, where the residuals are weighted with adjoint solutions that form sensitivities with regard to the error functional under consideration.

The DWR-method has been successfully applied to a large variety of problems including, for instance, flow simulations [BR06], aerodynamics [Har08] and structure dynamics [RS97]. Grätsch and Bathe [GB95] first applied goal-oriented error estimation to fluidstructure interaction problems. Sensitivity based adaptation for fsi-problems however are not well understood so far. In particular, the representation of the interface coupling conditions in the adjoint problems needs further understanding. Fick, Brummelen and Zee [FBZ10] analyzed the coupling of a potential flow with a lower dimensional boundary structure with regard to sensitivity based error estimation and pointed out the role of the coupling conditions for the adjoint solutions. Dunne [Dun07] and [DRR10] have first implementations of goal-oriented adaptation fluid-structure interactions with complex nonlinear models including two-dimensional fluid and solid problems. To our understanding, there is still no proper analysis of the adjoint problems appearing in sensitivity based error estimation or optimization of fluid-structure interaction problems. This is due to a lack of analysis on variational formulations for the coupled fluid-structure interaction problems.

The prerequisite for sensitivity based a posteriori error estimation is a variational formulation of the coupled problem. For this purpose, it is necessary, that the fsi-problem is given in a closed monolithic formulation. In this work, we use the Arbitrary Lagrangian Eulerian coordinates for modeling the coupled problem, which we will present in Section 3. Next, in Section 4 we derive a proper variational formulation for the monolithic fsi-problem in ALE coordinates. Here, we introduce specially adapted test-spaces with discontinuities across the coupling interface to obtain a proper variational formulation of the coupled fsi-problem.

The finite element discretization of this weak formulation is considered in Section 5 . In Section 6 we derive the dual weighted residual method for fsi-problems. The discontinuity of the test-spaces mentioned before will shift to the trial-spaces of the sensitivities. In Section 7 two benchmark problems are presented to analyze the efficiency of the resulting finite element scheme. We start with gathering basic notations and by presenting the underlying concepts of fluid-structure interaction problems in the following second section.

\section{Basic notations}

By $\Omega \subset \mathbb{R}^{d}$ with $d=2,3$ we denote a domain. This domain is split into a fluid-part $\Omega_{f}$ and into a solid part $\Omega_{s}$, each domains in $\mathbb{R}^{d}$. It holds $\Omega_{f} \cap \Omega_{s}=\emptyset$ and $\bar{\Omega}=\bar{\Omega}_{f} \cup \bar{\Omega}_{s}$. By $\Gamma_{i}:=\bar{\Omega}_{f} \cap \bar{\Omega}_{s}$ we denote the interface. In $\Omega_{f}$ the incompressible Navier-Stokes equations are given for the fluid's velocity $v_{f}: \Omega_{f} \rightarrow \mathbb{R}^{d}$ and pressure $p_{f}: \Omega_{f} \rightarrow \mathbb{R}$. In $\Omega_{s}$ an elastic material law is given to describe the solid's deformation $u_{s}: \Omega_{s} \rightarrow \mathbb{R}^{d}$. Figure 1 shows 

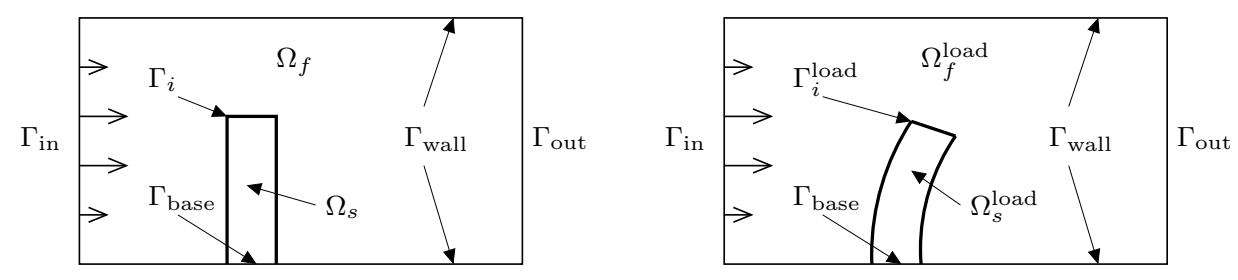

Figure 1: Typical configuration for a fsi-problem: flow around an elastic obstacle that is attached to the wall at $\Gamma_{\text {base }}$. Left: reference domain, right: configuration under load.

a typical configuration of a fsi-problem, where the flow encloses an elastic obstacle. The big challenge of fluid-structure interaction is the deformation of the domains $\Omega_{f}$ and $\Omega_{s}$ under load: the fluid's forces on the obstacle will cause a deformation $u_{s}$ of the solid via $T_{s}:=\mathrm{id}+u_{s}: \Omega_{s} \rightarrow \Omega_{s}^{\text {load }}$. Consequently, the flow domain will move along $\Omega_{f} \rightarrow \Omega_{f}^{\text {load }}$. When considering stationary problems, a new balance will be given by the loaded configuration $\bar{\Omega}=\bar{\Omega}_{f}^{\text {load }} \cup \bar{\Omega}_{s}^{\text {load }}$. The layout of this new configuration is not known a priori but unknown part of the solution.

On $\Omega$ we define by $L^{2}(\Omega)$ the Lebesgue space of square integrable functions on $\Omega$ with the $L^{2}$-inner product and norm:

$$
v, w \in L^{2}(\Omega): \quad(v, w)_{\Omega}:=\int_{\Omega} v w \mathrm{~d} x, \quad\|v\|_{\Omega}:=(v, v)_{\Omega}^{\frac{1}{2}}
$$

Usually, we skip the index $\Omega$ when referring to the whole domain and use $(\cdot, \cdot)_{x}:=(\cdot, \cdot)_{\Omega_{x}}$ with $x=s, f$ when referring to one of the subdomains. Further, by

$$
\langle v, w\rangle_{\Gamma}:=\int_{\Gamma} v w \mathrm{~d} s
$$

we denote the $L^{2}$-inner product along (parts of) the boundary. On the interface $\Gamma_{i}$ we use for abbreviation the notation $\langle\cdot, \cdot\rangle_{i}:=\langle\cdot, \cdot\rangle_{\Gamma_{i}}$. By $H^{1}(\Omega)$ we denote the space of $L^{2}(\Omega)$-functions with square-integrable weak derivatives in $L^{2}(\Omega)$. Finally, by $H_{0}^{1}(\Omega ; \Gamma)$ we denote the space of $H^{1}(\Omega)$-functions which have trace zero on (parts of) the boundary $\Gamma \subset \partial \Omega$.

\section{Modeling of 3D fluid-structure interaction}

In the flow domain $\Omega_{f}$ we consider the incompressible Navier-Stokes equations for modeling velocity $v_{f}$ and pressure $p_{f}$. Usually, this equation is formulated in the spatialcentered Eulerian coordinate framework, where velocity in pressure are given in fixed spatial points $x \in \Omega_{f}^{\text {load }}$. This means, that in the context of moving domains, the incompressible Navier-Stokes equations must be formulated in the unknown loaded configuration $\Omega_{f}^{\text {load}}$, shown in the right half of figure 1. In contrast, structural problems 
are usually modeled in the particle-centered Lagrangian coordinate framework in the left half of figure 1. By $u_{s}$ we denote the deformation of a fixed material point in the unloaded reference domain $x \in \Omega_{s}$. The coupling between fluid and structure is given by balancing conditions on the interface $\Gamma_{i}$. This interface however is either defined as $\Gamma_{i}:=\bar{\Omega}_{f} \cap \bar{\Omega}_{s}$ or $\Gamma_{i}^{\text {load }}:=\bar{\Omega}_{f}^{\text {load }} \cap \bar{\Omega}_{s}^{\text {load }}$, but the domain $\Omega_{f}^{\text {load }}$ for describing the fluid and the structure domain $\Omega_{s}$ do not have a common interface.

To deal with this dilemma and to derive a monolithic formulation we need to formulate the coupled problem in one matching coordinate framework. Here, we use the well established ALE coordinates, where the flow problem is mapped onto the reference domain $\Omega_{f}$ via a transformation $T_{f}: \Omega_{f} \rightarrow \Omega_{f}^{\text {load }}$ so that the two domains used for modeling the subproblems match with the interface $\Gamma_{i}$. Both subproblems are given on the sketch in the left half of figure 1.

ALE coordinates for modeling flow problems in moving domains have first been used by Hughes, Liu and Zimmermann [HLZ81]. Varies applications of fluid-structure interaction in ALE coordinates are found in the collections of Bungartz \& Schäfer [BS06] and [BS10]. The fundamental component for deriving the ALE formulation of fsi problems is the definition of the ALE mapping $T_{f}: \Omega_{f} \rightarrow \Omega_{f}^{\text {load }}$. Here it is common to write this transformation by using an artificial flow-domain deformation $u_{f}$ via $T_{f}(x):=x+$ $u_{f}(x)$. By requesting continuity $u_{f}=u_{s}$ on the interface $\Gamma_{i}$, an ALE mapping can be constructed in this easy manner. The artificial fluid's deformation $u_{f}$ is hence regarded as an extension of the physical solid's deformation $u_{s}$. Here, we simply use an harmonic extension for generating $u_{f}$ on $\Omega_{f}$. This easy extension is feasible for fsi-problems with moderate deformations but is known to have problems when considering very large deformation. Wick [Wic11] gives a survey of different extension techniques for the ALE mapping.

The coupled problem for the fluid's velocity $v_{f}$, pressure $p_{f}$ and artificial deformation $u_{f}$, as well as the solid's deformation $u_{s}$ is given by:

Problem 1 (Fluid-structure interaction in ALE coordinates).

$$
\begin{aligned}
& \rho_{f} J_{f} F_{f}^{-1} v_{f} \cdot \nabla v_{f}-\operatorname{div}\left(J_{f} \sigma_{f} F_{f}^{-T}\right)=J_{f} \rho_{f} f_{f} \\
& \left.\begin{array}{rl}
\operatorname{div}\left(J_{f} F_{f}^{-1} v_{f}\right) & =0 \\
-\Delta u_{f} & =0
\end{array}\right\} \text { in } \Omega_{f} \\
& \left.\begin{array}{rl}
-\operatorname{div}\left(J_{s} \sigma F_{s}^{-T}\right) & =\rho_{s} f_{s} \\
v_{s} & =0
\end{array}\right\} \text { in } \Omega_{s} \\
& \left.J_{s} \sigma_{s} F_{s}^{-T} n=J_{f} \sigma_{f} F_{f}^{-T} n\right) \quad(f) \\
& \left.v_{f}=v_{s} \quad\right\} \text { on } \Gamma_{i} \quad(g) \\
& u_{f}=u_{s} \quad \quad \quad(h) \\
& u_{f}=u_{s}=v_{s}=v_{f}=0 \quad \text { on } \Gamma^{\text {wall }}(i) \\
& v_{f}=v^{i n} \quad \text { on } \Gamma^{\text {in }} \quad(j) \\
& {\left[\rho_{f} \nu_{f} \nabla v_{f} F_{f}^{-1}-p_{f} I\right] n_{f}=0 \quad \text { on } \Gamma^{\text {out }}(k) \text {. }}
\end{aligned}
$$


Here, by $\sigma_{f}$ we denote the fluid's stress tensor in ALE coordinates, by $\sigma_{s}$ the tensor of the compressible St. Venant Kirchhoff material law:

$$
\sigma_{f}:=\rho_{f} \nu_{f}\left(\nabla v_{f} F_{f}^{-1}+F_{f}^{-T} \nabla v_{f}^{T}\right)-p_{f} I, \quad \sigma_{s}:=J_{s}^{-1} F_{s}\left(\lambda_{s}\left(\operatorname{tr} E_{s}\right) I+2 \mu_{s} E_{s}\right) F_{s}^{T},
$$

with the deformation gradients $F_{f}:=I+\nabla u_{f}$ and $F_{s}:=I+\nabla u_{s}$ and the strain tensor $E_{s}:=\frac{1}{2}\left(F_{s} F_{s}^{T}-I\right) . \quad J_{f}:=\operatorname{det}\left(F_{f}\right)$ and $J_{s}:=\operatorname{det}\left(F_{s}\right)$ are the deformation gradients determinants. By $\rho_{f}$ and $\rho_{s}$ we denote the fluid's and solid's density, by $\nu_{f}$ the fluid's kinematic viscosity and by $\lambda_{s}$ the Lamé coefficient and $\mu_{s}$ the shear modulus.

In $\Omega_{f}$ the incompressible Navier-Stokes equations transformed to ALE coordinates and in $\Omega_{s}$ an elastic material law of St. Venant Kirchhoff type is given. On the interface $\Gamma_{i}$, the two systems are coupled by demanding continuity of velocity (which is zero in $\Omega_{s}$ for stationary problems) and deformation as well as continuity of normal stresses between fluid and solid. On the outer boundaries of $\Omega$ we demand (for simplicity) homogenous Dirichlet conditions for the deformation, a no-slip condition on the wall boundary $\Gamma^{\text {wall }}$, a Dirichlet inflow condition on $\Gamma^{\text {in }}$ and the do-nothing outflow condition (see Heywood, Rannacher and Turek [HRT92]) on $\Gamma^{\text {out }}$.

Since velocity and deformation are defined on all $\Omega$ and are continuous over the interface $\Gamma_{i}$ we will shift this coupling conditions into the trial spaces by only searching continuous velocities and deformations $v, u: \Omega \rightarrow \mathbb{R}^{d}$ on the complete domain $\Omega$. Hence we skip the sub-notes "f" and "s". Further, since the deformation gradients $F_{f}$ and $F_{s}$ as well as the determinants $J_{f}$ and $J_{s}$ are likewise defined in both domains, we simply use $F$ and $J$ to denote both of them.

Remark 1 (Coupling conditions). The interface conditions on $\Gamma_{i}$ can be regarded symmetrically from both sides. We however consider the conditions on the interface to be read from left to right with Dirichlet data for $v$ and $u$ on $\Gamma_{i}$ in the fluid problem and Neumann data on $\Gamma_{i}$ for $u$ in the solid problem. This approach would also be typical for partitioned solution schemes of fsi-problems.

\section{Monolithic variational formulation for the fsi-problem}

As discussed before, velocity and deformation are continuous across the interface $\Gamma_{i}$. Hence, as trial spaces for a possible variational formulation of (1.a-1.k), we use:

$$
v \in v^{D}+V^{v}: V^{v}:=H_{0}^{1}\left(\Omega ; \Gamma^{\text {in }} \cup \Gamma^{\text {wall }}\right)^{d}, \quad u \in V^{u}: V^{u}:=H_{0}^{1}(\Omega ; \partial \Omega)^{d},
$$

where by $v^{D} \in H^{1}(\Omega)^{d}$ we denote an extension of the Dirichlet data on $\Gamma^{\text {in }}$ into the domain. The pressure $p$ is defined in $\Omega_{f}$ only:

$$
p \in V^{p}: V^{p}:=L^{2}\left(\Omega_{f}\right) .
$$

Coupling of the two momentum equations (1.a) and (1.d) is achieved by combining these two equations under one common continuous test-function $\phi \in V^{v}$. The interface 
flux condition (1.f) is then included due to integration by parts while the continuity condition (1.g) for $v$ is embedded in the trial space $V^{v}$ :

$$
\begin{aligned}
\left(J \sigma_{f} F^{-T}, \nabla \phi\right)_{f}+\left(J \sigma_{s} F^{-T}\right. & , \nabla \phi)_{s}=\left\langle n_{f} \cdot\left(J \sigma_{f} F^{-T}\right)+n_{s} \cdot\left(J \sigma_{s} F^{-T}\right), \phi\right\rangle \\
& -\left(\operatorname{div}\left(J \sigma_{f} F^{-T}\right), \phi\right)_{f}-\left(\operatorname{div}\left(J \sigma_{s} F^{-T}\right), \phi\right)_{s} \quad \forall \phi \in V^{v} .
\end{aligned}
$$

The equation for the extension of the deformation (1.c) is usually combined with equation (1.e), see e.g. Hron \& Turek or [HT06] or Richter \& Wick [RW10]. However, by multiplying these equations with a continuous test-function $\psi \in V^{u}$ (the trial space $V^{u}$ for the deformation has the proper Dirichlet values) artificial boundary terms appear due to integration by parts:

$$
(\nabla u, \nabla \psi)_{f}+(v, \psi)_{s}=\left\langle n_{f} \cdot \nabla u, \psi\right\rangle-(\Delta u, \psi)_{f}+(v, \psi)_{s} \quad \forall \psi \in V^{u} .
$$

This boundary term on $\Gamma_{i}$ introduces spurious feedback to the deformation in the solid domain $\Omega_{s}$ by spoiling the physical interface boundary conditions. In [DRR10] it is hence suggested to modify the variational formulation by subtracting this boundary term to:

$$
(\nabla u, \nabla \psi)_{f}+(v, \psi)_{s}-\left\langle n_{f} \cdot \nabla u, \psi\right\rangle=-(\Delta u, \nabla \psi)_{f}+(v, \psi)_{s} \quad \forall \psi \in V^{u} .
$$

While this formulation helps with removing the additional interface condition, the mathematical analysis of such boundary terms is awkward and will lead to difficulties when dealing with adjoint formulations. Another problem of both formulations is the different scaling of the two terms in the system matrix. While the discretization of the Laplacian behaves like $O(1)$, the entries in the solid part go like $O\left(h^{2}\right)$. This will deteriorate the performance of iterative solvers, see [Ric11]. A possible remedy which also deals with the artificial interface condition is a suitable scaling:

$$
(\nabla u, \nabla \psi)_{f}+\alpha(h)(v, \psi)_{s}=-(\Delta u, \psi)_{f}+\left\langle n_{f} \cdot \nabla u, \psi\right\rangle+\alpha(h)(v, \psi)_{s} \quad \forall \psi \in V^{u} .
$$

where $\alpha(h)=O\left(h^{-2}\right)$, see Richter \& Wick [RW10]. While this formulation numerically yields good results, the limit case $h \rightarrow 0$ is not understood. Further it still includes artificial boundary terms on the interface while lead to problems in adjoint formulations.

Hron [Hro] suggested to modify the system matrix to prevent artificial feedback to the solid problem. Here we follow this approach. In order to obtain the proper coupling condition with Dirichlet data for the extension, we modify the test-space $\hat{V}^{u}$ :

$$
\hat{V}^{u}:=\left\{\hat{\psi} \in L^{2}(\Omega): \hat{\psi}_{\mid \Omega_{f}} \in H_{0}^{1}\left(\Omega_{f} ; \partial \Omega_{f}\right), \hat{\psi}_{\mid \Omega_{s}} \in H_{0}^{1}\left(\Omega_{s} ; \partial \Omega_{s} \backslash \Gamma_{i}\right)\right\},
$$

such that all test-functions have trace zero on $\Gamma_{i}$ as seen from the fluid-domain $\Omega_{f}$ but have free values as seen from the solid domain $\Omega_{s}$. Hence, the test-space $\hat{V}^{u}$ is not continuous across $\Gamma_{i}$. By using this test-space for coupling (1.c) and (1.e) we prevent feedback from the artificial extension equation to the physical structure equation and it holds:

$$
(\nabla u, \nabla \hat{\psi})_{f}+(v, \hat{\psi})_{s}=-(\Delta u, \hat{\psi})_{f}+(v, \hat{\psi})_{s} \quad \forall \hat{\psi} \in \hat{V}^{u} .
$$

By combining (4) and (6) with the mass conservation equation (1.b) the variational formulation of the coupled fsi-problem is given by: 
Problem 2 (Variational formulation). With $X:=V^{v} \times V^{u} \times V^{p}$ and $\hat{X}:=V^{v} \times \hat{V}^{u} \times V^{p}$ find $U:=(v, u, p) \in U^{D}+X$ such that:

$$
\begin{aligned}
\left(\rho_{f} J F^{-1} v \cdot \nabla v, \phi\right)_{f}+\left(J \sigma_{f} F^{-T}, \nabla \phi\right)_{f}+\left(J \sigma_{s} F^{-T}, \nabla \phi\right)_{s}=0 & \forall \phi \in V^{v}, \\
(\nabla u, \nabla \hat{\psi})_{f}+(v, \hat{\psi})_{s}=0 & \forall \hat{\psi} \in \hat{V}^{u}, \\
\left(\operatorname{div}\left(J F^{-1} v\right), \xi\right)_{f}=0 & \forall \xi \in V^{p},
\end{aligned}
$$

with the stress-tensors and further parameters defined as in Problem 1 and where $U^{D}:=$ $\left(v^{D}, 0,0\right)$ is a suitable extension of the Dirichlet data into the domain.

\section{Finite Element Discretization for FSI Problems}

To ease the presentation, we write Problem 2 in a compact notation:

$$
U \in U^{D}+X, \quad a(U)(\hat{\Psi})=0 \quad \forall \hat{\Psi} \in \hat{X},
$$

where the semilinear form $a(\cdot)(\cdot)$ is composed according to $(7)$. For discretization we triangulate the domain $\Omega$ into a set of open hexahedrons in three or quadrilaterals in two spacial dimensions. This triangulation fulfills the usual regularity conditions, see [Cia78], which are loosened to include hanging nodes on faces and edges to allow for local mesh refinement, see [BR06] for a detailed presentation of the finite element discretization used. Here, we further demand, that the interface $\Gamma_{i}$ is resolved by mesh nodes $x_{i} \in \Omega_{i}$ and that for all (open) elements $K \in \Omega_{h}$ it holds $K \cap \Gamma_{i}=\emptyset$ such that only nodes, edges or faces of elements may coincide with the interface.

On the triangulation $\Omega_{h}$ we define the $r$-th order isoparametric finite element space

$$
V_{h}^{(r)}:=\left\{\phi_{h} \in H^{1}(\Omega):\left.\phi_{h}\right|_{K} \circ T_{K}^{-1} \in \mathcal{Q}^{(r)} \forall K \in \Omega_{h}\right\},
$$

where $T_{K}:(0,1)^{d} \rightarrow K$ is the isoparametric transformation in the polynomial space $\mathcal{Q}^{(r)}:=\operatorname{span}\left\{x_{1}^{\alpha_{1}} x_{2}^{\alpha_{2}} x_{3}^{\alpha_{3}}, 0 \leq \alpha_{i} \leq r\right\}$ from the reference element $\hat{K}:=(0,1)^{d}$ to the computational element. By $\left\{\phi_{h}^{(i)}, i=1, \ldots, N\right\}$ we denote a nodal basis of $V_{h}$. The discrete solution is then found in the space:

$$
U_{h} \in U_{h}^{d}+X_{h}, \quad X_{h}:=\left[V_{h}^{v}\right]^{d} \times\left[V_{h}^{u}\right]^{d} \times V_{h}^{p},
$$

with the proper modification of the base function in the corresponding nodes on the Dirichlet boundaries. As test-space we define:

$$
\hat{X}_{h}:=\left[V_{h}^{v}\right]^{d} \times\left[\hat{V}_{h}^{u}\right]^{d} \times V_{h}^{p},
$$

where in $\hat{V}_{h}^{u}$ we modify the nodal basis functions on the interface nodes $\Gamma_{i}$ by constraining their support to $\Omega_{f}$ according to Figure 2:

$$
x_{i} \in \Gamma_{i}: \quad \hat{\psi}_{i}(x)= \begin{cases}0 & x \in \Omega_{f}, \\ \psi_{i}(x) & x \in \Omega_{s} .\end{cases}
$$




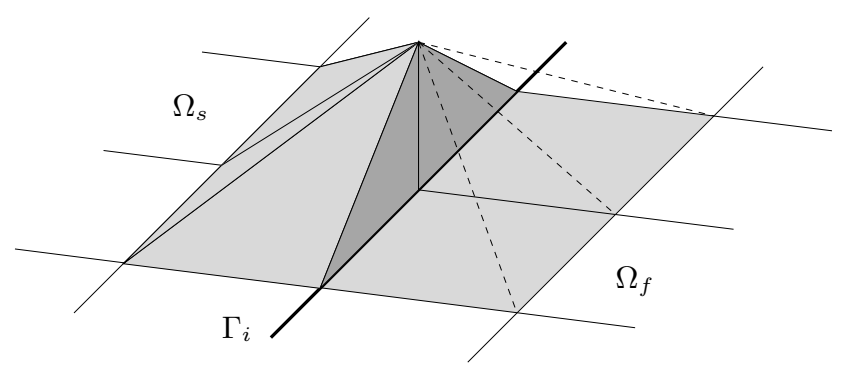

Figure 2: Modified basis functions in the fluid-domain along the interface $\Gamma_{i}$.

Remark 2 (Modified basis functions). We note, that the test-space $\hat{V}_{h}^{u}$ still has the same dimension as the trial-space $V_{h}^{u}$, such that the resulting system is quadratic. The discontinuous test-functions $\hat{\phi} \in \hat{V}_{h}^{u}$ are not discontinuous in a sense that two degrees of freedom are controlling one point, instead, every nodal basis functions on interface nodes is discontinuous.

\subsection{Local projection stabilization}

The incompressible Navier-Stokes system as basis of the fluid-structure interaction problem asks for approximation spaces fulfilling the inf-sup condition. Equal-order spaces like $\left[V_{h}^{(r)}\right]^{d}-V_{h}^{(r)}$ are not inf-sup stable, hence a modification of the semilinear form $a(\cdot)(\cdot)$ is necessary. Further, if the flow gets convection dominated, we need to stabilize the convective term. While the well established PSPG/SUPG method by Hughes, Franca and Balestra [HFB86] and Hughes and Brooks [HB82] exists for fluid-structure interaction problems, see Wall [Wa199], we refrain from using it due to the high numerical effort and the artificial coupling terms introduced by ensuring a consistent formulation. Instead, we use the LPS method as introduced by Becker and Braack [BB01, BB04]. For fluid-structure interaction in ALE coordinates, we add the stabilization form:

$$
\begin{aligned}
s_{\mathrm{lps}}\left(\bar{U}_{h}\right)\left(U_{h}, \Psi_{h}\right):=\sum_{K \in \Omega_{f}}\{( & \left(\alpha_{K} \bar{J} \bar{F}^{-1} \nabla \pi_{h} p_{h}, \bar{F}^{-1} \nabla \pi_{h} \xi_{h}\right)_{f} \\
& \left.\quad+\left(\delta_{K} \rho_{f} \bar{J}\left(\bar{F}^{-1} \bar{v}_{h}\right) \cdot \nabla \pi_{h} v_{h}, \rho_{f}\left(\bar{F}^{-1} \bar{v}_{h}\right) \cdot \nabla \pi_{h} \phi_{h}\right)_{f}\right\}
\end{aligned}
$$

where by $\pi_{h}:=i d-i_{h}^{(*)}$ with $i_{h}^{(*)}: V_{h} \rightarrow V_{h}^{(*)}$ we denote the fluctuation operator with regard to a stable space like $V_{h}^{(*)}:=V_{h}^{(r-1)}$. The parameters $\alpha_{K}$ and $\delta_{K}$ depend on the mesh Peclet number and are defined by

$$
\alpha_{K}=\delta_{K}=\delta_{0}\left(\frac{\rho_{f} \nu_{f}}{h_{K}^{2}}+\frac{\rho_{f}\|\bar{v}\|_{\infty, K}}{h_{K}}\right)^{-1}
$$

See Braack and Lube [BL09] for a detailed discussion on the local projection stabilization and [Ric11] for a first application to fluid-structure interaction problems. Finally, we 
arrive at the following stabilized variational formulation of the fluid-structure interaction problem:

Problem 3 (Stabilized finite element discretization). Find $U_{h} \in U_{h}^{D}+X_{h}$ with $X_{h}:=$ $\left[V_{h}^{v}\right]^{d} \times\left[V_{h}^{u}\right]^{d} \times V_{h}^{p}$, such that:

$$
a\left(U_{h}\right)\left(\hat{\Psi}_{h}\right)+s_{l p s}\left(U_{h}\right)\left(U_{h}, \hat{\Psi}_{h}\right)=0 \quad \forall \hat{\Psi}_{h} \in \hat{X}_{h},
$$

with $\hat{X}_{h}:=\left[V_{h}^{v}\right]^{d} \times\left[\hat{V}_{h}^{u}\right]^{d} \times V_{h}^{p}$ and where the semilinear form $a(\cdot)(\cdot)$ is defined by $(7)$ and the stabilization form $s_{l p s}(\cdot)(\cdot, \cdot)$ by $(9)$.

\subsection{Solution scheme}

System (11) is a highly nonlinear complex system of partial differential equations. For its solution, we treat the equations by a Newton's method looking for iterates $U_{h}^{(i)} \rightarrow U_{h}$. In each step of the Newton scheme, the update $W_{h}^{(i)} \in X_{h}$ with $U_{h}^{(i+1)}:=U_{h}^{(i)}+W_{h}^{(i)}$ is defined by the linear system

$$
\begin{aligned}
a^{\prime}\left(U_{h}^{(i)}\right)\left(W_{h}^{(i)}, \hat{\Psi}_{h}\right)+s_{\text {lps }}\left(U_{h}^{(i)}\right) & \left(W_{h}^{(i)}, \hat{\Psi}_{h}\right)= \\
& -a\left(U_{h}^{(i)}\right)\left(\hat{\Psi}_{h}\right)-s_{\text {lps }}\left(U_{h}^{(i)}\right)\left(U_{h}^{(i)}, \hat{\Psi}_{h}\right) \quad \forall \hat{\Psi}_{h} \in \hat{X}_{h},
\end{aligned}
$$

where $a_{h}^{\prime}\left(U_{h}\right)\left(W_{h}, \hat{\Psi}_{h}\right)$ is the directional derivative of $a_{h}^{\prime}\left(U_{h}\right)\left(\hat{\Psi}_{h}\right)$ at point $U_{h}$ in direction $W_{h}$. Even though a calculation of this derivative would be possible with finite differences or automatic differentiation, for reasons of stability we usually analytically evaluate all derivatives. Note that we neglect all nonlinear couplings in the stabilization form $s_{\mathrm{lps}}(\cdot)(\cdot, \cdot)$. This is justified by observation of the resulting convergence rates. By $A_{h}$ we denote the system matrix of the linear problems in the Newton iteration (12):

$$
A_{h}:=\left(A_{i j}\right)_{i j=1}^{N}, A_{i j}=a^{\prime}\left(U_{h}\right)\left(\Phi_{h}^{j}, \hat{\Psi}_{h}^{i}\right)+s_{\mathrm{lps}}\left(U_{h}\right)\left(\Phi_{h}^{j}, \hat{\Psi}_{h}^{i}\right), \quad \Phi_{j} \in X_{h}, \hat{\Psi}_{i} \in \hat{X}_{h} .
$$

For solving the linear systems we employ a GMRES iteration, preconditioned by a geometric multigrid solver. As smoother for this multigrid iteration we use a partitioning iteration which separately tackles the solid and fluid problem on each level. Here, the correct treatment of the interface coupling is essential: while the solid sub-problem is considered with a Neumann coupling, the fluid sub-problem is coupled with Dirichlet data. See [Ric11] for details on the solution schemes. Theoretical results on using partitioned approaches for smoothing a multigrid iteration are found in Brummelen et al [BZB08].

\section{The dual weighted residual method and adaptive mesh refinement}

The main goal of this section is to employ the dual weighted residual method (DWR) for the adaptive solution of fsi-problems. It provides a general framework for the derivation 
of goal-oriented a posteriori error estimates and gives simultaneously mesh adaption criteria for the Galerkin discretization of (nonlinear) variational problems. For the DWR method, a monolithic variational formulation of the fluid-structure Problem 1 is indispensable. Before concentrating on specific aspects of fluid-structure interaction, we derive the abstract setting of the DWR method following Becker \& Rannacher [BR01].

\subsection{The dual weighted residual method}

We consider a PDE given in variational formulation and its discretization with stabilized finite elements:

$$
\begin{array}{rlc}
U \in U^{D}+X & a(U)(\hat{\Psi})=0 & \hat{\Psi} \in \hat{X} \\
U_{h} \in U_{h}^{D}+X_{h} & a\left(U_{h}\right)\left(\hat{\Psi}_{h}\right)+s_{\mathrm{lps}}\left(U_{h}\right)\left(U_{h}, \hat{\Psi}_{h}\right)=0 & \hat{\Psi}_{h} \in \hat{X}_{h} .
\end{array}
$$

By $E_{h}:=U-U_{h}$ we denote the error. We aim at estimating this error in an output functional $J: X \rightarrow \mathbb{R}$. We introduce adjoint problems which yield sensitivities $\hat{Z} \in$ $\hat{Z}^{D}+\hat{X}$ and $\hat{Z}_{h} \in \hat{Z}_{h}^{D}+\hat{X}_{h}$ with regard to the functional considered:

$$
\begin{aligned}
a^{\prime}(U)(\Phi, \hat{Z}) & =J^{\prime}(U)(\Phi) & & \Phi \in X, \\
a^{\prime}\left(U_{h}\right)\left(\Phi_{h}, \hat{Z}_{h}\right)+s_{\mathrm{lps}}\left(U_{h}\right)\left(\Phi_{h}, \hat{Z}_{h}\right) & =J^{\prime}\left(U_{h}\right)\left(\Phi_{h}\right) & & \Phi_{h} \in X_{h} .
\end{aligned}
$$

By $\hat{E}_{h}^{*}:=\hat{Z}-\hat{Z}_{h}$ we denote the discretization error of the adjoint solution.

Remark 3. Note, that the system matrix of the adjoint problem (15) is given by the transposed Jacobian in the primal Newton scheme (12). The adjoint problem is always linear and since the system matrix is already available, the numerical effort to calculate the adjoint solution is comparable to one additional Newton step.

Further note, that in the adjoint problems the role of trial- and test-spaces is switched. Hence, the sensitivities are found in the space $Z \in \hat{X}$ with discontinuities across the coupling interface.

The following theorem is a slight modification of Becker \& Rannacher [BR01], considering non-conformity due to the stabilization term added to the discrete formulation:

Theorem 1 (Error representation for the fluid-structure interaction problem). For the functional error between $U$ and $U_{h}$ it holds:

$$
\begin{aligned}
J(U)-J\left(U_{h}\right) & =\frac{1}{2}\left\{J^{\prime}\left(U_{h}\right)\left(U-i_{h} U\right)-a^{\prime}\left(U_{h}\right)\left(U-i_{h} U, \hat{Z}_{h}\right)\right\} \\
- & \frac{1}{2} a\left(U_{h}\right)\left(\hat{Z}-\hat{i}_{h} \hat{Z}\right)+\frac{1}{2} s_{l p s}\left(U, \hat{Z}_{h}\right)+\frac{1}{2} s_{l p s}\left(U_{h}, \hat{Z}\right)+\mathcal{R}^{(3)}\left(E_{h}, E_{h}^{(*)}\right),
\end{aligned}
$$

where $\mathcal{R}^{(3)}$ is a remainder of third order in the errors $E_{h}$ and $\hat{E}_{h}^{(*)}$ :

$$
\begin{array}{r}
\mathcal{R}^{(3)}:=\frac{1}{2} \int_{0}^{1}\left\{J^{\prime \prime \prime}\left(U_{h}+s E_{h}\right)\left(E_{h}, E_{h}, E_{h}\right)-3 a^{\prime \prime}\left(U_{h}+s E_{h}\right)\left(E_{h}, E_{h}, E_{h}^{*}\right)\right. \\
\left.-a^{\prime \prime \prime}\left(U_{h}+s E_{h}\right)\left(E_{h}, E_{h}, E_{h}, \hat{Z}_{h}+s \hat{E}_{h}^{*}\right)\right\} s(s-1) d s .
\end{array}
$$


Proof: We introduce the Lagrange functionals $L: X \times \hat{X} \rightarrow \mathbb{R}$ and $L_{h}: X_{h} \times \hat{X}_{h} \rightarrow \mathbb{R}$ :

$$
L(\Phi, \hat{\Psi}):=J(\Phi)-a(\Phi)(\hat{\Psi}), \quad L_{h}\left(\Phi_{h}, \hat{\Psi}_{h}\right):=L_{h}\left(\Phi_{h}, \hat{\Psi}_{h}\right)-s_{\operatorname{lps}}\left(U_{h}\right)\left(\Phi_{h}, \hat{\Psi}_{h}\right) .
$$

Let $U$ and $U_{h}$ be the primal solutions of (14), $\hat{Z}$ and $\hat{Z}_{h}$ the adjoint solutions of (15). Hence, it holds $L^{\prime}(U, \hat{Z})(\Phi, \hat{\Psi})=0$ for all $\Phi \in X$ and $\hat{\Psi} \in \hat{X}$ (analogously for the discrete functions $\left.L_{h}^{\prime}\right)$. For the error $J(U)-J\left(U_{h}\right)=L(U, Z)-L_{h}\left(U_{h}, Z_{h}\right)$ we obtain:

$$
J(U)-J\left(U_{h}\right)=\int_{0}^{1} \frac{d}{d s} L\left(U_{h}+s E_{h}, \hat{Z}_{h}+s \hat{E}_{h}^{*}\right) \mathrm{d} s+s_{\mathrm{lps}}\left(U_{h}\right)\left(U_{h}, \hat{Z}_{h}\right)
$$

Approximation with the trapezoidal rule yields

$$
J(U)-J\left(U_{h}\right)=\frac{1}{2} L^{\prime}\left(U_{h}, \hat{Z}_{h}\right)\left(E_{h}, \hat{E}_{h}^{*}\right)+s_{\mathrm{lps}}\left(U_{h}\right)\left(U_{h}, \hat{Z}_{h}\right)+\mathcal{R}^{(3)},
$$

with the remainder $\mathcal{R}^{(3)}$ of the trapezoidal rule as detailed above. The second evaluation point $L^{\prime}(U, \hat{Z})\left(E_{h}, \hat{E}_{h}^{*}\right)=0$ is zero, since $\{U, \hat{Z}\} \in X \times \hat{X}$ is the stationary point of the Lagrange functional $L$ and the discretization is conforming with $X_{h} \times \hat{X}_{h} \subset X \times \hat{X}$. By expanding $L^{\prime}$ and insertion of missing stabilization terms we obtain

$$
\begin{aligned}
& J(U)-J\left(U_{h}\right)=\frac{1}{2}\left\{J^{\prime}\left(U_{h}\right)\left(E_{h}\right)-a^{\prime}\left(U_{h}\right)\left(E_{h}, \hat{Z}_{h}\right)-s_{\mathrm{lps}}\left(U_{h}\right)\left(E_{h}, \hat{Z}_{h}\right)\right\} \\
& -\frac{1}{2}\left\{a\left(U_{h}\right)\left(\hat{E}_{h}^{*}\right)+s_{\mathrm{lps}}\left(U_{h}\right)\left(U_{h}, \hat{E}_{h}^{*}\right)\right\}+\frac{1}{2}\left\{s_{\mathrm{lps}}\left(U_{h}\right)\left(U, \hat{Z}_{h}\right)+s_{\mathrm{lps}}\left(U_{h}\right)\left(U_{h}, \hat{Z}\right)\right\}+\mathcal{R}^{(3)}
\end{aligned}
$$

Use of Galerkin orthogonality yields the theorem.

Remark 4 (Discussion of the remainder terms). The variational formulation of the fluid-structure interaction problem 7 is highly nonlinear. Since the inverse of the transformation gradient $F^{-1}$ appears in the formulations the derivatives of the Lagrange functional will not vanish, for example, it holds with $F(u)=I+\nabla u$ :

$$
\begin{aligned}
& \left.\frac{d}{d s} F^{-1}(u+s w)\right|_{s=0}=F^{-1} \nabla w F^{-1} \\
& \left.\frac{d^{2}}{d^{2} s} F^{-1}(u+s w)\right|_{s=0}=2 F^{-1} \nabla w F^{-1} \nabla w F^{-1} \\
& \left.\frac{d^{3}}{d^{3} s} F^{-1}(u+s w)\right|_{s=0}=6 F^{-1} \nabla w F^{-1} \nabla w F^{-1} \nabla w F^{-1} .
\end{aligned}
$$

Evaluated in the error-direction $w:=E_{h}$, this remainder is of third oder in the energy error $\left\|\nabla E_{h}\right\|$. The boundness depends on the regularity of the transformation gradient $F$. When $J=\operatorname{det}(F) \rightarrow 0$, the remainder term may get large. Within the structure problem, $J$ is a measure for the compressibility of the material, $J \rightarrow 0$ or $J \rightarrow \infty$ is prohibited by physical principals. Within the flow problem however, $J$ is a measure for the quality of the ALE mapping. This mapping is known to degenerate, see Wick [Wic11] when considering large deformations. 


\subsection{Error estimation and mesh adaptation}

To evaluate the error identity (16) we need to neglect the remainder term. This is justifiable, if the regularity of the problem is sufficient which - in the context of fsi - is the case, if the deformation is moderate. It remains to approximate the interpolation errors $U-i_{h} U$ and $\hat{Z}-\hat{i}_{h} \hat{Z}$. Several approximations have been discussed. In the classical approach by Becker \& Rannacher [BR95] the residuals in the error identity (16) are partially integrated to result in the strong residual of the partial differential equation of type:

$$
J(U)-J\left(U_{h}\right)=\sum_{K \in \Omega_{h}}-\left(\nabla \cdot\left(J \sigma_{f} F^{-T}\right), \hat{Z}-\hat{i}_{h} \hat{Z}\right)_{K}+\left\langle n \cdot\left(J \sigma_{f} F^{-T}\right), \hat{Z}-\hat{i}_{h} \hat{Z}\right\rangle_{\partial K}+\ldots
$$

This transformation would allow to estimate with Cauchy-Schwarz and to approximate the integration errors $\left\|\hat{Z}-\hat{i}_{h} \hat{Z}\right\|_{K}$ by finite difference approximations. We however refrain from using this transformation since the evaluation of the strong residuals is very costly due to the nonlinearities and the second spatial derivatives involved. For using the weak formulation to evaluate the residuals, the adjoint solution $\hat{Z}$ must be approximated with higher accuracy. Relying on super-approximation properties we construct this "better" approximation by using a local interpolation into a higher order finite element space $i_{2 h}^{(2)}: V_{h}^{(r)} \rightarrow V_{2 h}^{(2 r)}$ and approximate:

$$
U-i_{h} U \approx i_{2 h}^{(2)} U_{h}-U_{h}, \quad \hat{Z}-\hat{i}_{h} \hat{Z} \approx \hat{i}_{2 h}^{(2)} \hat{Z}_{h}-\hat{Z}_{h} .
$$

Even though this approximation cannot be justified from a rigorous mathematical point of view since super-approximation results are not at hand for the equations under consideration, this approach is widely and successfully used in different applications, see [BR06, KR10]. In our context, we need to assure, that for higher order reconstruction of the adjoint solution we do not violate the special interface coupling-condition $\hat{V}_{2 h}^{(2 r)} \subset \hat{V}$. Primal and adjoint solution further appear in the the stabilization terms in (16). These will also be replaced by the higher order reconstruction. This approach is legitimate, since the stabilization consists of projection errors only and hence is local. Finally, we estimate the error by:

$$
\begin{aligned}
J(U)-J\left(U_{h}\right) \approx \eta & :=\frac{1}{2}\left\{J^{\prime}\left(U_{h}\right)\left(i_{2 h}^{(2)} U_{h}-U_{h}\right)-a^{\prime}\left(U_{h}\right)\left(i_{2 h}^{(2)} U_{h}-U_{h}, \hat{Z}_{h}\right)\right\} \\
& -\frac{1}{2} a\left(U_{h}\right)\left(\hat{i}_{2 h}^{(2)} \hat{Z}_{h}-\hat{Z}_{h}\right)+\frac{1}{2} s_{\mathrm{lps}}\left(i_{2 h}^{(2)} U_{h}, \hat{Z}_{h}\right)+\frac{1}{2} s_{\mathrm{lps}}\left(U_{h}, \hat{i}_{2 h}^{(2)} \hat{Z}_{h}\right) .
\end{aligned}
$$

In Section 7, numerical examples will demonstrate the accuracy of this approximation. For mesh adaptation we need to localize the error to local error indicators $\eta_{i}$ satisfying:

$$
\left|J(U)-J\left(U_{h}\right)\right| \approx|\eta| \leq \sum_{i=1}^{N} \eta_{i}, \quad \eta_{i} \geq 0 .
$$

It is well known, that a simple element-wise localization of the integrals in (18) does not yield the proper order of convergence and would lead to false adaptation of the meshes. 

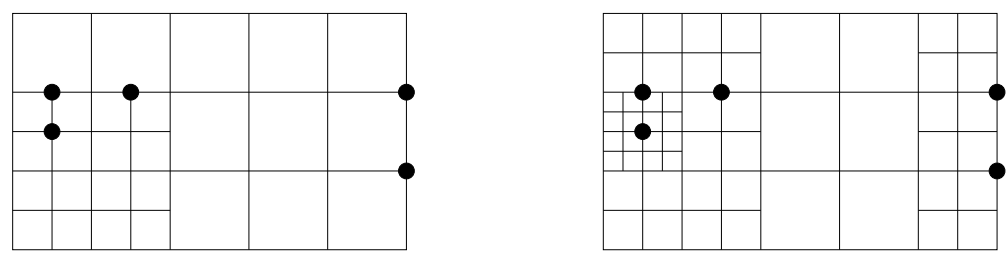

Figure 3: Refinement of the mesh. 5 nodes are picked for refinement. This results in the refinement of 9 elements.

Instead we use Galerkin orthogonality to introduce another interpolation into a coarser space $\hat{i}_{2 h}: \hat{X} \rightarrow \hat{X}_{2 h} \subset \hat{X}_{h} \subset \hat{X}$. To ease the presentation we only discuss the primal residual term in the estimator:

$$
\begin{aligned}
J(U)-J\left(U_{h}\right) & \approx-a\left(U_{h}\right)\left(\hat{Z}-\hat{i}_{h} \hat{Z}\right)=-a\left(U_{h}\right)\left(\left(\hat{Z}-\hat{i}_{h} \hat{Z}\right)-\hat{i}_{2 h}\left(\hat{Z}-\hat{i}_{h} \hat{Z}\right)\right) \\
& =-a\left(U_{h}\right)\left(\left(\hat{Z}-\hat{i}_{2 h} \hat{Z}\right)-\hat{i}_{h}\left(\hat{Z}-\hat{i}_{2 h} \hat{Z}\right)\right) \\
& \approx-a\left(U_{h}\right)\left(\left(\hat{i}_{2 h}^{(2)} \hat{Z}_{h}-\hat{i}_{2 h} \hat{Z}_{h}\right)-\hat{i}_{h}\left(i_{2 h}^{(2)} \hat{Z}_{h}-\hat{i}_{2 h} \hat{Z}_{h}\right)\right) .
\end{aligned}
$$

By restricting these residual terms to local, node-wise contributions, we yield the proper local approximation order.

For mesh adaptation we simply refine all those nodes with above average estimator contribution:

$$
\text { refine node } x_{i} \in \Omega_{h} \quad \Leftrightarrow \quad \eta_{i} \geq \alpha_{\text {ref }}\left(\frac{1}{N} \sum_{j=1}^{N} \eta_{j}\right),
$$

with some parameter $\alpha_{\text {ref }} \approx 1$. Refining a node means refining all elements $K \in \Omega_{h}$ which have this node as a corner $x_{i} \in \bar{K}$. If the node $x_{i}$ is a hanging node, we only refine the coarse elements, where $x_{i}$ touches the midpoint of a face or edge. See Figure 3 for an example.

At this point, we want to stress the special character of the coupling between the fluid and the solid problem. In the primal variational formulation test-functions with discontinuities across the interface are used. By dualization, these discontinuities are shifted into the trial-space in the adjoint formulation (15). Hence, the proper sensitivities $\hat{Z} \in \hat{X}_{h}$ have jumps at the interface $\Gamma_{i}$. In Figure 4 we show an adjoint solution which represents the sensitivity to estimating the lift-coefficient of an elastic obstacle within a flow domain. This visualization is taken from the FSI-1 benchmark analyzed as first example in Section 7.

\section{Numerical Results}

In this section, we study two problems and show the efficiency of our proposed adaptive method. First we consider the stationary FSI-1 benchmark as proposed by Hron, Turek et. al. in $\left[\mathrm{HTM}^{+} 10\right]$. In this two-dimensional problem, a laminar flow around a circular 

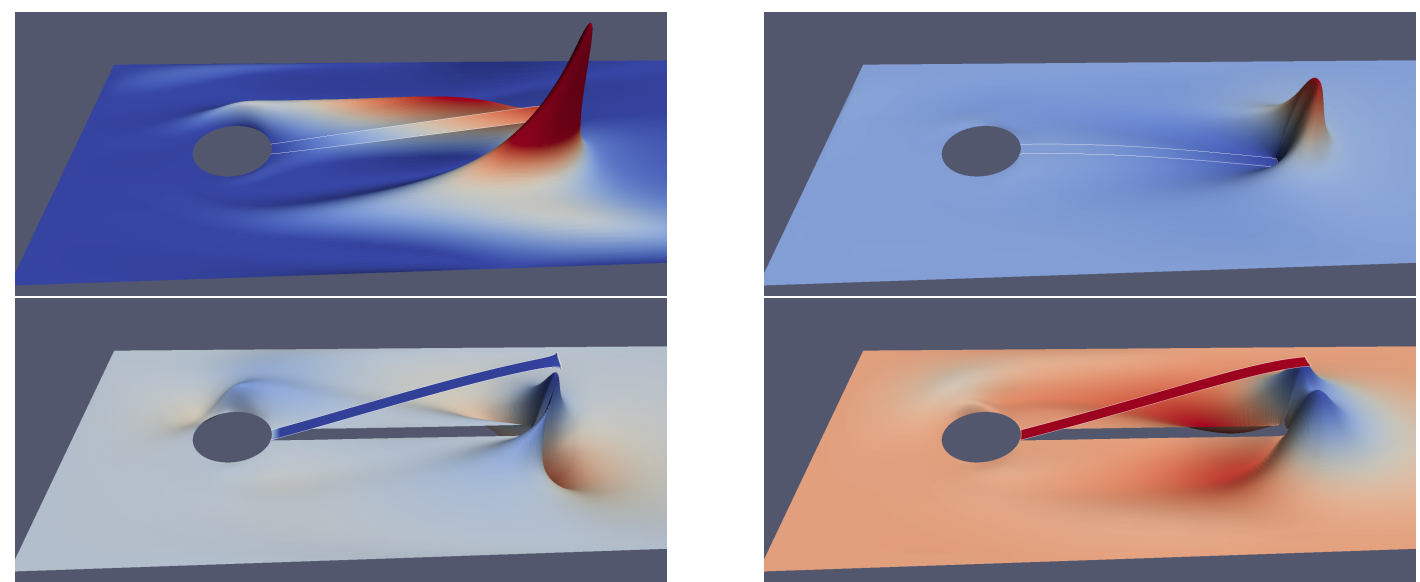

Figure 4: Sensitivity with regard to the lift-evaluation. The adjoint solution components belonging to the deformation (lower row) have discontinuities at the interface $\Gamma_{i}$. From upper left to lower right: $x$-velocity, $y$-velocity, $x$-deformation and $y$-deformation.

obstacle with an attached elastic beam is considered. Object of interest is the drag coefficient of the obstacle as well as the deformation of one point on the tip of the beam.

Secondly, we analyze as three-dimensional benchmark problem the laminar flow over an elastic obstacle attached to the wall. As quantities of interest we again evaluate the deformation in a point within the obstacle and the drag coefficient.

\subsection{The FSI-1 benchmark problem}

Firstly, we consider the stationary FSI benchmark problem FSI 1 [BS06, BS10]. Here, the laminar flow around a cylinder, with an attached elastic bar is simulated. Figure 5 shows a sketch of the configuration.

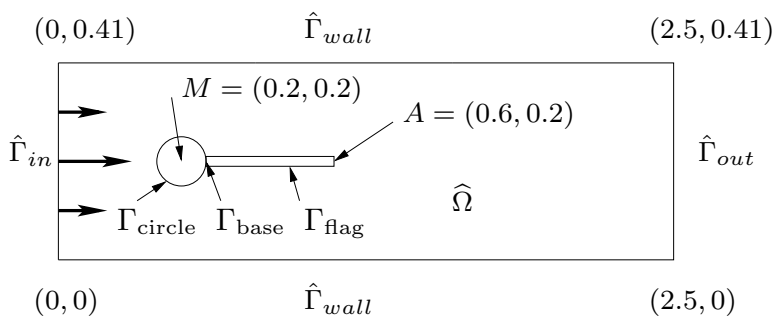

Figure 5: Flow around cylinder with elastic beam with circle-center $C=(0.2,0.2)$ and radius $r=0.05$. 


\begin{tabular}{r|rrrr}
\hline dof's & drag & lift & $u^{x}(A)$ & $u^{y}(A)$ \\
\hline 1440 & 14.45584 & 0.730486 & $2.1656 \cdot 10^{-5}$ & $8.8801 \cdot 10^{-4}$ \\
5360 & 14.27783 & 0.784080 & $2.2134 \cdot 10^{-5}$ & $8.8474 \cdot 10^{-4}$ \\
20640 & 14.28727 & 0.766030 & $2.2604 \cdot 10^{-5}$ & $8.2650 \cdot 10^{-4}$ \\
80960 & 14.29135 & 0.765160 & $2.2647 \cdot 10^{-5}$ & $8.2469 \cdot 10^{-4}$ \\
320640 & 14.29304 & 0.764960 & $2.2662 \cdot 10^{-5}$ & $8.2214 \cdot 10^{-4}$ \\
1276160 & 14.29362 & 0.764861 & $2.2671 \cdot 10^{-5}$ & $8.2067 \cdot 10^{-4}$ \\
5091840 & 14.29383 & 0.764812 & $2.2676 \cdot 10^{-5}$ & $8.1982 \cdot 10^{-4}$ \\
\hline extra & 14.29395 & 0.764761 & $2.2683 \cdot 10^{-5}$ & $8.1860 \cdot 10^{-4}$ \\
\hline
\end{tabular}

Table 1: Results for the FSI-1 benchmark on uniform meshes using piece-wise quadratic finite elements.

Problem configuration Three benchmark problems have been proposed. We limit the considerations to the stationary FSI-1 case with the following set of physical parameters

$$
\rho_{f}=\rho_{s}=1000, \quad \nu_{f}=10^{-3}, \quad \mu_{s}=5 \cdot 10^{5}, \quad \lambda_{s}=2 \cdot 10^{6}, \quad \bar{U}=0.2
$$

The compressible St. Venant-Kirchhoff material is used to describe the elastic structure. As quantity of interest, we regard the horizontal and vertical deflection of the structure in the point $A=(0.6,0.2)$ on the tip of the bar, as well as the drag- and lift- coefficient of the complete obstacle (the rigid circle and the elastic flag):

$$
\begin{aligned}
J_{\mathrm{drag}}(U) & =\int_{S}\left(J \sigma_{f} F^{-T}\right) n_{f} e_{1} \mathrm{~d} s & J_{\text {lift }}(U) & =\int_{S}\left(J \sigma_{f} F^{-T}\right) n_{f} e_{2} \mathrm{~d} s \\
J_{\mathrm{x}}(U) & =u_{1}(A) & J_{\mathrm{y}}(U) & =u_{2}(A),
\end{aligned}
$$

where $e_{i}:=\left(\delta_{i 1}, \delta_{i 2}\right)$ are the Cartesian unit vectors and $S:=\Gamma_{\text {flag }} \cup \Gamma_{\text {circle }}$.

Higher order evaluation of the surface integrals To evaluate the lift- and dragcoefficient we transform the surface integrals into volume integrals. This is well-known to yield better approximation results, see e.g. Braack \& Richter [BR06]. Here, we start by using the coupling condition (1.f) on the interface $\Gamma_{i}$ :

$$
\begin{aligned}
J_{\text {drag }}(U) & =\int_{\Gamma_{\text {circle }}}\left(J \sigma_{f} F^{-T}\right) n_{f} e_{1} \mathrm{~d} s+\int_{\Gamma_{i}}\left(J \sigma_{f} F^{-T}\right) n_{f} e_{1} \mathrm{~d} s \\
& =\int_{\Gamma_{\text {circle }}}\left(J \sigma_{f} F^{-T}\right) n_{f} e_{1} \mathrm{~d} s-\int_{\Gamma_{i}}\left(J \sigma_{s} F^{-T}\right) n_{s} e_{1} \mathrm{~d} s \\
& =\int_{\Gamma_{\text {circle }}}\left(J \sigma_{f} F^{-T}\right) n_{f} e_{1} \mathrm{~d} s+\int_{\Gamma_{\text {base }}}\left(J \sigma_{s} F^{-T}\right) n_{s} e_{1} \mathrm{~d} s
\end{aligned}
$$

where in the last step we used the relation $-\operatorname{div}\left(J \sigma_{s} F^{-T}\right)=0$. Hence, we can compute the drag as the surface integral over the complete circle and by using different tensors 
in different parts of the boundary. Following [BR06], this integral can be expressed as:

$$
J_{\text {drag }}(U)=a(U)(\hat{\chi})
$$

with $a(\cdot)(\cdot)$ given as in (8) and with the special non-conforming test-function $\hat{\chi}:=$ $\left(0, \chi_{1}, 0,0,0\right) \notin \hat{X}$ :

$$
\hat{\chi}_{1}=\left\{\begin{aligned}
1 & : x \in \Gamma_{\text {circle }} \cup \Gamma_{\text {base }} \\
\text { extended to } 0 & : x \notin \Gamma_{\text {circle }} \cup \Gamma_{\text {base }}
\end{aligned}\right.
$$

Obtaining Reference values of the FSI-1 benchmark problem This benchmark problem is well analyzed in the collection [BS06] and the follow-up publication [BS10]. In Table 1 we gather the results of a simulation on uniform meshes using quadratic finite elements. By extrapolation we identify the reference values as indicated in Table 2. These values are in very good agreement with the results published by Turek et. al. [THR $\left.{ }^{+} 10\right]$.

\begin{tabular}{lcc}
\hline functional & reference value & accuracy \\
\hline drag & 14.29395 & $\pm 5 \cdot 10^{-5}$ \\
lift & 0.76480 & $\pm 5 \cdot 10^{-5}$ \\
$x$-deformation & $2.2680 \cdot 10^{-5}$ & $\pm 5 \cdot 10^{-9}$ \\
$y$-deformation & $8.190 \cdot 10^{-4}$ & $\pm 5 \cdot 10^{-7}$ \\
\hline
\end{tabular}

Table 2: Reference values for the FSI-1 benchmark.

Error estimation and results on locally refined meshes For error estimation with the dual weighted residual method we need to approximate the adjoint problems. In the case of the horizontal deflection, the adjoint problems are given by:

$$
\hat{Z} \in \hat{X} \quad a^{\prime}(U)(\Phi, \hat{Z})=\psi_{1}(A), \quad \forall \Phi:=\left(\xi, \phi_{1}, \phi_{2}, \psi_{1}, \psi_{2}\right) \in X .
$$

This problem is solved with homogenous Dirichlet data on the parts of the boundary $\Gamma_{\text {in }} \cup \Gamma_{\text {wall }} \cup \Gamma$ where $U$ has Dirichlet data and with homogenous Neumann data on the remaining boundary parts. With $J_{\text {drag }}$ as described in (19), we obtain:

$$
\hat{Z} \in \hat{X} \quad a^{\prime}(U)(\Phi, \hat{Z})=a^{\prime}(U)(\Phi, \hat{\chi}) \quad \forall \Phi \in X .
$$

This right hand side can be regarded as the extension of non-homogenous Dirichlet values $\hat{\chi}$ into the domain and we can reformulate the adjoint problems for drag and lift functionals as

$$
\hat{Z} \in \hat{Z}^{D}+\hat{X} \quad a^{\prime}(U)(\Phi, \hat{Z})=0 \quad \forall \Phi \in X,
$$

where the extension of Dirichlet data $\hat{Z}^{D}$ is given by

$$
Z^{D}:=\left(0, z_{1}^{D}, 0,0,0\right), \quad z_{1}^{D}= \begin{cases}1 & \text { on } \Gamma_{\text {circle }} \cup \Gamma_{\text {base }} \\ 0 & \text { else. }\end{cases}
$$




\begin{tabular}{|c|c|c|c|c|c|c|}
\hline dof's & $J_{\text {drag }}\left(U_{h}\right)$ & error (abs) & error (rel) & $J_{\text {lift }}\left(U_{h}\right)$ & error (abs) & error (rel) \\
\hline 1440 & 15.2491 & $9.55 \cdot 10^{-1}$ & $6.68 \cdot 10^{-2}$ & 0.76670 & $1.98 \cdot 10^{-3}$ & $2.49 \cdot 10^{-3}$ \\
\hline 5360 & 14.4587 & $1.65 \cdot 10^{-1}$ & $1.15 \cdot 10^{-2}$ & 0.79498 & $3.02 \cdot 10^{-2}$ & $3.97 \cdot 10^{-2}$ \\
\hline 20640 & 14.3340 & $4.01 \cdot 10^{-2}$ & $2.80 \cdot 10^{-3}$ & 0.77339 & $8.59 \cdot 10^{-3}$ & $1.13 \cdot 10^{-2}$ \\
\hline 80960 & 14.3060 & $1.21 \cdot 10^{-2}$ & $8.46 \cdot 10^{-4}$ & 0.76714 & $2.34 \cdot 10^{-3}$ & $3.08 \cdot 10^{-3}$ \\
\hline 320640 & 14.2982 & $4.25 \cdot 10^{-3}$ & $2.97 \cdot 10^{-4}$ & 0.76547 & $6.75 \cdot 10^{-4}$ & $8.88 \cdot 10^{-4}$ \\
\hline 1276160 & 14.2956 & $1.65 \cdot 10^{-3}$ & $1.15 \cdot 10^{-4}$ & 0.76501 & $2.12 \cdot 10^{-4}$ & $2.79 \cdot 10^{-4}$ \\
\hline 5091840 & 14.2947 & $7.54 \cdot 10^{-4}$ & $5.27 \cdot 10^{-5}$ & 0.76486 & $6.12 \cdot 10^{-5}$ & $8.05 \cdot 10^{-5}$ \\
\hline dof's & $J_{\mathrm{x}}\left(U_{h}\right)$ & error & $J_{\mathrm{y}}\left(U_{h}\right)$ & error & & \\
\hline 1440 & $2.7378 \cdot 10^{-5}$ & $4.70 \cdot 10^{-6}$ & $2.07 \cdot 10^{-1}$ & $2.458 \cdot 10^{-4}$ & $-5.73 \cdot 10^{-4}$ & $7.00 \cdot 10^{-1}$ \\
\hline 5360 & $2.3502 \cdot 10^{-5}$ & $8.22 \cdot 10^{-7}$ & $3.62 \cdot 10^{-2}$ & $7.966 \cdot 10^{-4}$ & $-0.22 \cdot 10^{-5}$ & $2.69 \cdot 10^{-2}$ \\
\hline 20640 & $2.2919 \cdot 10^{-5}$ & $2.39 \cdot 10^{-7}$ & $1.05 \cdot 10^{-2}$ & $8.191 \cdot 10^{-4}$ & $5.13 \cdot 10^{-8}$ & $6.26 \cdot 10^{-4}$ \\
\hline 80960 & $2.2763 \cdot 10^{-5}$ & $8.35 \cdot 10^{-8}$ & $3.68 \cdot 10^{-3}$ & $8.232 \cdot 10^{-4}$ & $4.19 \cdot 10^{-6}$ & $5.12 \cdot 10^{-3}$ \\
\hline 320640 & $2.2708 \cdot 10^{-5}$ & $2.83 \cdot 10^{-8}$ & $1.25 \cdot 10^{-3}$ & $8.223 \cdot 10^{-4}$ & $3.31 \cdot 10^{-6}$ & $4.04 \cdot 10^{-3}$ \\
\hline 1276160 & $2.2689 \cdot 10^{-5}$ & $9.53 \cdot 10^{-9}$ & $4.20 \cdot 10^{-4}$ & $8.210 \cdot 10^{-4}$ & $2.03 \cdot 10^{-6}$ & $2.48 \cdot 10^{-3}$ \\
\hline 5091840 & $2.2684 \cdot 10^{-5}$ & $4.41 \cdot 10^{-9}$ & $1.94 \cdot 10^{-4}$ & $8.201 \cdot 10^{-4}$ & $1.09 \cdot 10^{-6}$ & $1.33 \cdot 10^{-3}$ \\
\hline
\end{tabular}

Table 3: Simulation of the FSI-1 benchmark problem on uniform meshes using piece-wise linear finite elements. Bold lines: error below $0.1 \%$.

In Figure 4 in Section 6 we show the adjoint solutions with regard to the lift evaluation. Here one clearly sees the discontinuity at the interface.

In Tables 4 we list the results obtained by local mesh refinement for the drag- and lift-coefficient as well as for the deflection in the tip of the flag in horizontal and vertical direction. For each mesh we indicate the number of unknowns, the functional value, absolute and relative error measured against the reference value from 1, the DWRestimator value and the effectivity

$$
J_{\text {eff }}\left(U_{h}\right):=\frac{\eta}{J^{\text {ref }}-J\left(U_{h}\right)}
$$

of the estimator. For comparison, in Table 3 we list the corresponding values obtained on globally refined meshes. All these computations are performed using piece-wise linear finite elements. In all tables we have highlighted the meshes where the relative error first goes below $0.1 \%$ accuracy. While in the case of the more regular force functionals $J_{\text {drag }}$ and $J_{\text {lift }}$ no significant savings are gained at this level, for the two deflection functionals $J_{\mathrm{x}}$ and $J_{\mathrm{y}}$ this error level is reached with ten times less degrees of freedom. For the vertical deflection, calculations on uniform meshes could not reach that tolerance level at all.

Further we want to highlight the effectivity of the error estimator indicated in the last columns of Table 4. Apart from the calculations belonging to the vertical deflection, all error estimates are highly accurate and able to predict the error. Considering point-wise functionals which lack regularity $\left(J_{\mathrm{x}}, J_{\mathrm{y}} \notin H^{-1}(\Omega)\right)$ and strictly speaking would require regularization, the estimates show a larger variation. This holds especially for the more delicate case of the vertical deflection $J_{\mathrm{y}}(U)$. 


\begin{tabular}{|c|c|c|c|c|c|}
\hline dof's & $J_{\text {drag }}\left(U_{h}\right)$ & error (abs) & error (rel) & est & eff \\
\hline 1440 & 15.2491 & $9.55 \cdot 10^{-1}$ & $6.68 \cdot 10^{-2}$ & $1.19 \cdot 10^{-0}$ & 0.12 \\
\hline 4550 & 14.4639 & $1.70 \cdot 10^{-1}$ & $1.19 \cdot 10^{-2}$ & $2.58 \cdot 10^{-1}$ & 1.52 \\
\hline 15340 & 14.3393 & $4.53 \cdot 10^{-2}$ & $3.17 \cdot 10^{-3}$ & $6.00 \cdot 10^{-2}$ & 1.32 \\
\hline 49520 & 14.3085 & $1.46 \cdot 10^{-2}$ & $1.02 \cdot 10^{-3}$ & $1.74 \cdot 10^{-2}$ & 1.19 \\
\hline 163300 & 14.2992 & $5.20 \cdot 10^{-3}$ & $3.64 \cdot 10^{-4}$ & $5.66 \cdot 10^{-3}$ & 1.09 \\
\hline 490540 & 14.2961 & $2.16 \cdot 10^{-3}$ & $1.51 \cdot 10^{-4}$ & $2.17 \cdot 10^{-3}$ & 1.00 \\
\hline 1323550 & 14.2949 & $9.64 \cdot 10^{-4}$ & $6.74 \cdot 10^{-5}$ & $8.99 \cdot 10^{-4}$ & 0.93 \\
\hline dof's & $J_{\text {lift }}\left(U_{h}\right)$ & error (abs) & error (rel) & est & eff \\
\hline 1440 & 0.76674 & $1.98 \cdot 10^{-3}$ & $2.61 \cdot 10^{-3}$ & $2.72 \cdot 10^{-2}$ & 13.7 \\
\hline 4690 & 0.79402 & $2.93 \cdot 10^{-2}$ & $3.86 \cdot 10^{-2}$ & $2.55 \cdot 10^{-3}$ & 0.09 \\
\hline 13950 & 0.77598 & $1.12 \cdot 10^{-2}$ & $1.47 \cdot 10^{-2}$ & $9.46 \cdot 10^{-3}$ & 0.84 \\
\hline 42610 & 0.76792 & $3.16 \cdot 10^{-3}$ & $4.16 \cdot 10^{-3}$ & $2.24 \cdot 10^{-3}$ & 0.71 \\
\hline 135360 & 0.76583 & $1.07 \cdot 10^{-3}$ & $1.41 \cdot 10^{-3}$ & $8.07 \cdot 10^{-4}$ & 0.75 \\
\hline 430650 & 0.76521 & $4.50 \cdot 10^{-4}$ & $5.92 \cdot 10^{-4}$ & $3.33 \cdot 10^{-4}$ & 0.74 \\
\hline 1301450 & 0.76497 & $1.97 \cdot 10^{-4}$ & $2.59 \cdot 10^{-4}$ & $1.58 \cdot 10^{-4}$ & 0.80 \\
\hline dof's & $J_{\mathrm{x}}\left(U_{h}\right)$ & error (abs) & error (rel) & est & eff \\
\hline 1440 & $2.73781 \cdot 10^{-5}$ & $4.70 \cdot 10^{-6}$ & $2.07 \cdot 10^{-1}$ & $5.64 \cdot 10^{-6}$ & 1.20 \\
\hline 4560 & $2.33783 \cdot 10^{-5}$ & $6.98 \cdot 10^{-7}$ & $3.08 \cdot 10^{-2}$ & $1.24 \cdot 10^{-6}$ & 1.78 \\
\hline 12740 & $2.29398 \cdot 10^{-5}$ & $2.60 \cdot 10^{-7}$ & $1.15 \cdot 10^{-2}$ & $2.79 \cdot 10^{-7}$ & 1.07 \\
\hline 37110 & $2.27795 \cdot 10^{-5}$ & $9.95 \cdot 10^{-8}$ & $4.39 \cdot 10^{-3}$ & $1.08 \cdot 10^{-7}$ & 1.09 \\
\hline 104780 & $2.27189 \cdot 10^{-5}$ & $3.89 \cdot 10^{-8}$ & $1.72 \cdot 10^{-3}$ & $4.66 \cdot 10^{-8}$ & 1.20 \\
\hline 230840 & $2.26956 \cdot 10^{-5}$ & $1.56 \cdot 10^{-8}$ & $6.88 \cdot 10^{-4}$ & $2.00 \cdot 10^{-8}$ & 1.28 \\
\hline 535970 & $2.26877 \cdot 10^{-5}$ & $7.70 \cdot 10^{-9}$ & $3.40 \cdot 10^{-4}$ & $9.28 \cdot 10^{-9}$ & 1.21 \\
\hline dof's & $J_{\mathrm{y}}\left(U_{h}\right)$ & error (abs) & error (rel) & est & eff \\
\hline 1440 & $2.4577 \cdot 10^{-4}$ & $-5.73 \cdot 10^{-4}$ & $7.00 \cdot 10^{-1}$ & $2.51 \cdot 10^{-4}$ & -0.44 \\
\hline 3850 & $7.5595 \cdot 10^{-4}$ & $-6.31 \cdot 10^{-5}$ & $7.70 \cdot 10^{-2}$ & $7.35 \cdot 10^{-5}$ & -1.16 \\
\hline 11670 & $8.1076 \cdot 10^{-4}$ & $-8.24 \cdot 10^{-6}$ & $1.01 \cdot 10^{-2}$ & $1.27 \cdot 10^{-5}$ & -1.54 \\
\hline 35560 & $8.1574 \cdot 10^{-4}$ & $-3.26 \cdot 10^{-6}$ & $3.98 \cdot 10^{-3}$ & $5.16 \cdot 10^{-6}$ & -1.58 \\
\hline 102580 & $8.1905 \cdot 10^{-4}$ & $5.00 \cdot 10^{-7}$ & $6.11 \cdot 10^{-4}$ & $2.07 \cdot 10^{-6}$ & 0.41 \\
\hline 229240 & $8.2007 \cdot 10^{-4}$ & $1.07 \cdot 10^{-6}$ & $1.31 \cdot 10^{-3}$ & $2.99 \cdot 10^{-7}$ & 0.28 \\
\hline 532150 & $8.1957 \cdot 10^{-4}$ & $5.70 \cdot 10^{-7}$ & $6.96 \cdot 10^{-4}$ & $1.20 \cdot 10^{-7}$ & 0.21 \\
\hline
\end{tabular}

Table 4: 2d problem FSI-1: adaptive finite elements. From top to bottom: drag, lift, horizontal and vertical deflection.

\subsection{D fluid-structure interaction}

Problem configuration Finally, we present numerical simulations of a three dimensional test-case. In the domain $\Omega:=(0,1.5) \times(0,0.4) \times(-0.4,0.4)$ an elastic structure 


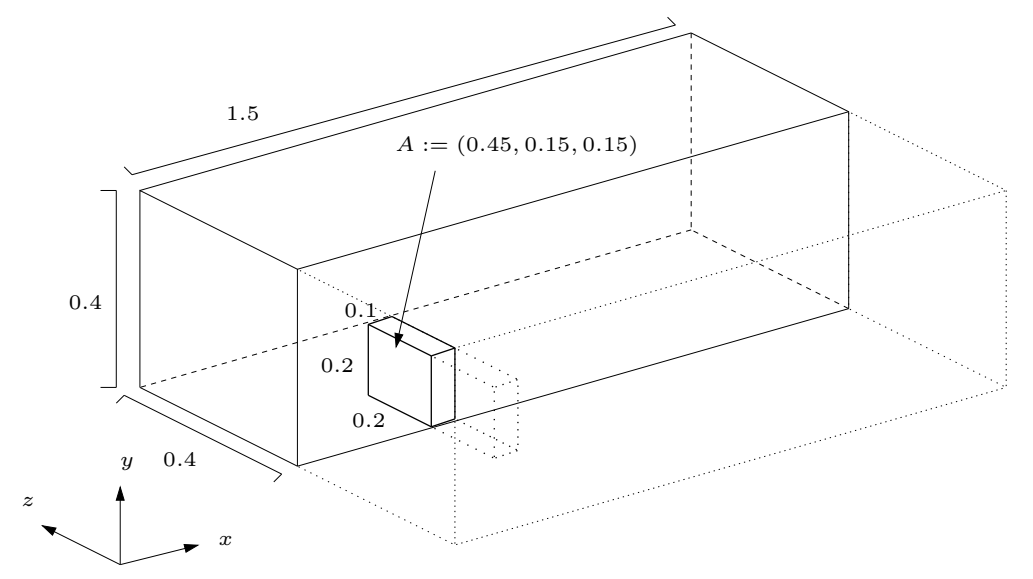

Figure 6: Configuration of the three-dimensional test-case. Domain and solution are symmetric in $z$-direction.

$\Omega_{s}:=(0.4,0.5) \times(0,0.2) \times(-0.2,0.2)$ is inscribed. The problem is considered to be symmetric in the $x-y$-plane. Hence, we run the simulation only in one half of the domain. On the inflow boundary $\Gamma_{\text {in }}$, a parabolic velocity profile is given as Dirichlet condition

$$
v_{\text {in }}=\frac{0.3}{0.2^{2} \cdot 0.4^{2}}\left(\begin{array}{c}
y(0.4-y)\left(0.4^{2}-z^{2}\right) \\
0 \\
0
\end{array}\right),
$$

with peak velocity $v_{\max }=0.3$. On the inner symmetry plane, we prescribe $v \cdot n=0$ as Dirichlet condition, on the outflow boundary $\Gamma_{\text {out }}$ the do-nothing condition and a no-slip condition on the remaining walls. The solid is fixed by a Dirichlet condition on the bottom and deformation in normal-direction is prohibited on the symmetry-base:

$$
\begin{aligned}
v=v_{\text {in }} \text { on } \Gamma_{\text {in }}, n \cdot v=0 \text { on } \Gamma_{\text {sym }}, J \sigma_{f} F^{-T}=0 \text { on } \Gamma_{\text {out }}, v=0 \text { on } \Gamma_{\text {wall }}, \\
\\
u=0 \text { on } \Gamma_{\text {base }} \cap \Gamma_{\text {wall }}, n \cdot u=0 \text { on } \Gamma_{\text {base }} \cap \Gamma_{\text {sym }} .
\end{aligned}
$$

The fluid is incompressible with $\rho_{f}=1000$ and $\nu_{f}=10^{-3}$. The solid's Lamé coefficients are $\nu_{s}=0.4$ and $\mu_{s}=5 \cdot 10^{5}$. With an average inflow velocity of 0.2 , and an obstacle of size 0.2 , the Reynolds number is $R e=40$. For LPS stabilization, we set the parameter in (10) to $\delta_{0}=0.2$.

As quantities of interest, we measure the deformation of the obstacle in measure the deformation at the coordinate $A=(0.45,0.15,0.15)$ close to the outer corner of the structure, as well as the force of the fluid on the structure in the dominant flow direction:

$$
J_{\mathrm{x}}(U):=e_{1} \cdot u(A), \quad J_{\operatorname{drag}}(U):=\int_{\Gamma_{i}} n \cdot\left(J \sigma_{f} F^{-T}\right) e_{1} \mathrm{~d} s .
$$




\begin{tabular}{r|rrr|rrr}
\hline dof's & $J_{\text {drag }}\left(U_{h}\right)$ & error (abs) & error $($ rel $)$ & $J_{\mathrm{x}}\left(U_{h}\right)$ & error $($ abs $)$ & error $(\mathrm{rel})$ \\
\hline 2975 & 1.5249 & $1.98 \cdot 10^{-1}$ & $1.49 \cdot 10^{-1}$ & $4.9337 \cdot 10^{-5}$ & $9.90 \cdot 10^{-6}$ & $1.67 \cdot 10^{-1}$ \\
18711 & 1.4763 & $1.49 \cdot 10^{-1}$ & $1.13 \cdot 10^{-1}$ & $5.5686 \cdot 10^{-5}$ & $3.55 \cdot 10^{-6}$ & $6.00 \cdot 10^{-2}$ \\
131495 & 1.4038 & $7.68 \cdot 10^{-2}$ & $5.79 \cdot 10^{-2}$ & $5.8529 \cdot 10^{-5}$ & $7.11 \cdot 10^{-7}$ & $1.20 \cdot 10^{-2}$ \\
$\mathbf{9 8 3 ~ 3 6 7}$ & 1.3563 & $2.93 \cdot 10^{-2}$ & $2.20 \cdot 10^{-2}$ & $\mathbf{5 . 9 0 7 5} \cdot \mathbf{1 0}^{-\mathbf{5}}$ & $\mathbf{1 . 6 5} \cdot \mathbf{1 0}^{-\mathbf{7}}$ & $\mathbf{2 . 7 9} \cdot \mathbf{1 0}^{-\mathbf{3}}$ \\
$\mathbf{7 6 0 0 ~ \mathbf { 6 7 5 }}$ & $\mathbf{1 . 3 3 8 0}$ & $\mathbf{1 . 1 0} \cdot \mathbf{1 0}^{-\mathbf{2}}$ & $\mathbf{8 . 2 9 \cdot \mathbf { 1 0 } ^ { - 3 }}$ & $5.9202 \cdot 10^{-5}$ & $3.80 \cdot 10^{-8}$ & $6.41 \cdot 10^{-4}$ \\
\hline \hline
\end{tabular}

Table 5: Convergence history of the three dimensional fsi test-case using piece-wise linear finite elements. The bold lines indicate, that an error of under $1 \%$ has been reached.

For the surface integrals it holds like in the two-dimensional case:

$$
\begin{aligned}
J_{\operatorname{drag}}(U) & =\int_{\Gamma_{i}} n_{f} \cdot\left(J \sigma_{f} F^{-T} e_{1}\right) \mathrm{d} s=-\int_{\Gamma_{i}} n_{s} \cdot\left(J \sigma_{s} F^{-T} e_{1}\right) \mathrm{d} s \\
& =\int_{\Gamma_{\text {base }}} n_{s} \cdot\left(J \sigma_{s} F^{-T} e_{1}\right) \mathrm{d} s=a(U)\left(\hat{\chi}_{1}\right) .
\end{aligned}
$$

Hence, instead of evaluating surface integrals on $\Gamma_{i}$, drag- and lift-coefficients can be measured on the fixed boundary $\Gamma_{\text {base }}$, where the solid hits the outer boundary of $\Omega$. Further, as explained above, the surface integral is transformed into a three-dimensional residual term evaluated using a non-conforming test-function $\hat{\chi}_{1}$.

Obtaining Reference values of the $\mathbf{3 d}$ benchmark problem For obtaining reference values we estimate the two error quantities on a sequence of meshes using uniform refinement. Table 5 gathers the results. By extrapolating, using the values on the finest three meshes, we fix the reference values in Table 6 .

\begin{tabular}{lcc}
\hline functional & reference value & accuracy \\
\hline drag & 1.327 & $\pm 1 \cdot 10^{-2}$ \\
$x$-deflection & $5.924 \cdot 10^{-5}$ & $\pm 1 \cdot 10^{-7}$ \\
\hline
\end{tabular}

Table 6: Reference values for the three dimensional benchmark problem.

We believe this values to be exact to a relative error of at most $1 \%$. It was not possible to achieve more reliable results by using piece-wise quadratic finite elements. Due to lacking regularity of the solution introduced by the structure's reentrant corners, no gain in convergence order was observed.

Error estimation and results on locally refined meshes In Tables 7 we present the results of computations using adaptive mesh refinement. For both error functionals, the drag coefficient in the upper table and the horizontal deflection in the lower one, we 


\begin{tabular}{|c|c|c|c|c|c|}
\hline dof's & $J_{\text {drag }}\left(U_{h}\right)$ & error (abs) & error (rel) & estimator & eff \\
\hline 2975 & 1.5249 & $1.98 \cdot 10^{-1}$ & $1.49 \cdot 10^{-1}$ & $2.11 \cdot 10^{-0}$ & 10.6 \\
\hline 11039 & 1.4883 & $1.61 \cdot 10^{-1}$ & $1.21 \cdot 10^{-1}$ & $6.66 \cdot 10^{-1}$ & 4.13 \\
\hline 57365 & 1.4083 & $8.13 \cdot 10^{-2}$ & $6.13 \cdot 10^{-2}$ & $7.21 \cdot 10^{-2}$ & 0.89 \\
\hline 245469 & 3534 & $2.64 \cdot 10^{-2}$ & $1.99 \cdot 10$ & $2.61 \cdot 10^{-2}$ & 0.99 \\
\hline 946379 & 3380 & $1.13 \cdot 10^{-2}$ & 8.52 & 9.3 & 0.83 \\
\hline 3691793 & 1.3325 & $5.54 \cdot 10^{-3}$ & $4.17 \cdot 10^{-}$ & $5.15 \cdot 10^{-3}$ & 0.93 \\
\hline dof's & $J_{\mathrm{x}}\left(U_{h}\right)$ & error (abs) & error (rel) & & eff \\
\hline 2975 & $337 \cdot 10^{-5}$ & $-9.90 \cdot 10^{-6}$ & $1.67 \cdot 10^{-1}$ & $-5.68 \cdot 10^{-5}$ & 5.73 \\
\hline 11039 & $5.6083 \cdot 10^{-5}$ & $-3.16 \cdot 10^{-6}$ & $5.33 \cdot 10^{-2}$ & $-1.87 \cdot 10^{-5}$ & 5.91 \\
\hline 41265 & $5.8405 \cdot 10^{-5}$ & $-8.36 \cdot 10^{-7}$ & $1.41 \cdot 10^{-2}$ & $-6.96 \cdot 10^{-6}$ & 8.33 \\
\hline 159481 & $109 \cdot 10^{-5}$ & $-1.31 \cdot 10^{-7}$ & $2.21 \cdot 10^{-3}$ & $-1.06 \cdot 10^{-6}$ & 8.09 \\
\hline 708575 & $5.9204 \cdot 10^{-5}$ & $-3.59 \cdot 10^{-8}$ & $6.06 \cdot 10^{-4}$ & $-2.96 \cdot 10^{-7}$ & 8.24 \\
\hline 2830779 & $5.9280 \cdot 10^{-5}$ & $3.99 \cdot 10^{-8}$ & $6.74 \cdot 10^{-4}$ & $-3.13 \cdot 10^{-7}$ & -- \\
\hline
\end{tabular}

Table 7: 3d problem: adaptive finite elements. Upper table: estimating the force functional $J_{\text {drag. }}$. Lower table: estimating the horizontal $J_{\mathrm{x}}$. The bold lines indicate an error below $1 \%$.

indicate the number of total unknowns, the functional value, relative and absolute error values as compared to the reference values in Table 6 , the result of the dual weighted residual estimator and the effectivity index, see (20). We have highlighted the calculations where the error first reaches $1 \%$ accuracy. For both functionals, this tolerance level can be reached with about 10 times less degrees of freedom compared to using uniform meshes. As in the two dimensional case, the error estimates regarding the drag coefficient are highly accurate, with an effectivity (20) very close to 1 . In the case of the singular point-functional $J_{\mathrm{x}}$ we overestimate the error by a factor of nearly 10 . On the finest mesh, the reference value for the deflection is not accurate enough to compare the estimator value.

Figure 7 shows a visualization of a numerical solution belonging to Table 7. Here, adaptation is driven in order to optimize the functional value $J_{\mathrm{x}}\left(U_{h}\right)$. The figure shows the final mesh. Deformation of the structure is scaled by 100 for better visualization.

\section{Conclusion}

Focus of this work is the accurate analysis of the weak formulation used for deriving the monolithic formulation of the fluid-structure interaction problem. By taking discontinuities in the test-space, the proper interface coupling condition was considered. This special test-cases lead to discontinuous adjoint solutions. At least for regular settings like the drag or lift coefficient, the dual weighted residual method yields highly accurate estimates for the error. These estimates can be used to generate efficient meshes for 


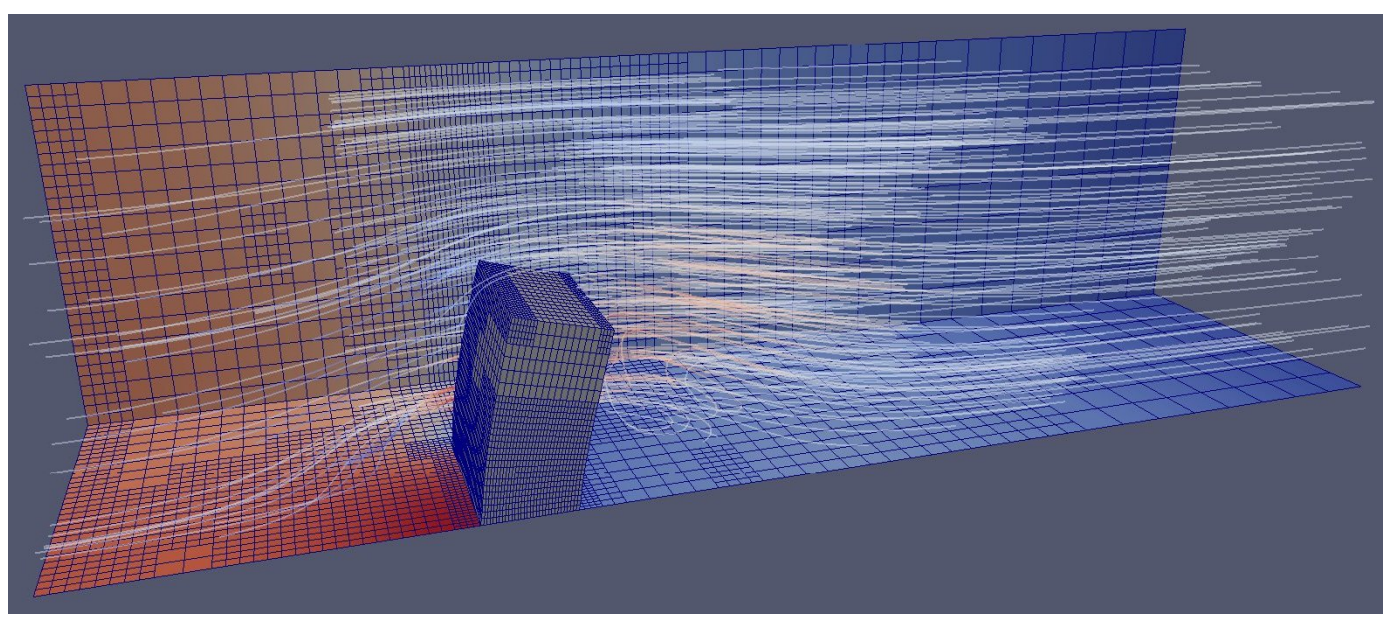

Figure 7: Sketch of the numerical solution on locally refined meshes. Domain is cut at the symmetry plane.

obtaining functional values. In particular when dealing with complex three dimensional problems the possible saving due to accurate adaptive meshes are immense.

The variational formulation derived for goal-oriented error estimation has further impact on all numerical schemes where adjoint problems are involved. In particular, the correct representation of the adjoint interface coupling conditions is of importance when considering optimization of fluid-structure interaction.

\section{References}

[AO97] M. Ainsworth and J. T. Oden. A posteriori error estimation in finite element analysis. Computer Methods in Applied Mechanics and Engineering, 142(12):1-88, 1997.

[BB01] R. Becker and M. Braack. A finite element pressure gradient stabilization for the Stokes equations based on local projections. Calcolo, 38(4):173-199, 2001.

[BB04] R. Becker and M. Braack. A two-level stabilization scheme for the NavierStokes equations. In et. al. M. Feistauer, editor, Numerical Mathematics and Advanced Applications, ENUMATH 2003, pages 123-130. Springer, 2004.

[BL09] M. Braack and G. Lube. Finite elements with local projection stabilization for incompressible flow problems. Journal of Computational Mathematics, 27:116-147, 2009.

[BR95] R. Becker and R. Rannacher. Weighted a posteriori error control in FE 
methods. In et al. H. G. Bock, editor, ENUMATH'97. World Sci. Publ., Singapore, 1995.

[BR01] R. Becker and R. Rannacher. An optimal control approach to a posteriori error estimation in finite element methods. In A. Iserles, editor, Acta Numerica 2001, volume 37, pages 1-225. Cambridge University Press, 2001.

[BR06] M. Braack and T. Richter. Solutions of 3D Navier-Stokes benchmark problems with adaptive finite elements. Computers and Fluids, 35(4):372-392, May 2006.

[BS06] H.-J. Bungartz and M. Schäfer, editors. Fluid-Structure Interaction. Modelling, Simulation, Optimisation, volume 53 of Lecture Notes in Computational Science and Engineering. Springer, 2006. ISBN-10: 3-540-34595-7.

[BS10] H.-J. Bungartz and M. Schäfer, editors. Fluid-Structure Interaction II. Modelling, Simulation, Optimisation. Lecture Notes in Computational Science and Engineering. Springer, 2010.

[BZB08] E.H. van Brummelen, K.G. van der Zee, and R. de Borst. Space/time multigrid for a fluid-structure-interaction problem. Applied Numerical Mathematics, 58(12):1951-1971, 2008.

[Cia78] P.G. Ciarlet. Finite Element Methods for Elliptic Problems. North-Holland, Amsterdam, 1978.

[DRR10] T. Dunne, R. Rannacher, and T. Richter. Numerical simulation of fluidstructure interaction based on monolithic variational formulations. In G.P. Galdi and R. Rannacher, editors, Comtemporary Challenges in Mathematical Fluid Mechanics. World Scientific, Singapore, 2010.

[Dun07] T. Dunne. Adaptive Finite Element Approximation of Fluid-Structure Interaction Based on Eulerian and Arbitrary Lagrangian-Eulerian Variational Formulations. PhD thesis, University of Heidelberg, 2007. urn:nbn:de:bsz:16opus-79448.

[EEHJ95] K. Eriksson, D. Estep, P. Hansbo, and C. Johnson. Introduction to adaptive methods for differential equations. In A. Iserles, editor, Acta Numerica 1995, pages 105-158. Cambridge University Press., 1995.

[FBZ10] P.W. Fick, E.H. van Brummelen, and K.G. van der Zee. On the adjointconsistent formulation of interface conditions in goal-oriented error estimation and adaptivity for fluid-structure interaction. Computer Methods in Applied Mechanics and Engineering, 199:3369-3385, 2010.

[GB95] T. Grätsch and K.J. Bathe. Goal-oriented error estimation in the analysis of fluid flows with structural interactions. Computer Methods in Applied Mechanics and Engineering, 2006:5673-5684, 195. 
[Har08] Ralf Hartmann. Multitarget error estimation and adaptivity in aerodynamic flow simulations. SIAM J. Sci. Comput., 31(1):708-731, 2008.

[HB82] T.J.R. Hughes and A.N. Brooks. Streamline upwind Petrov Galerkin formulations for convection dominated flows with particular emphasis on the incompressible Navier-Stokes equation. Computer Methods in Applied Mechanics and Engineering, 32:199-259, 1982.

[HFB86] T.J.R. Hughes, L.P. Franca, and M. Balestra. A new finite element formulation for computational fluid dynamics: V. circumvent the Babuska-Brezzi condition: A stable Petrov-Galerkin formulation for the Stokes problem accommodating equal order interpolation. Computer Methods in Applied Mechanics and Engineering, 59:89-99, 1986.

[HLZ81] T.J.R. Hughes, W.K. Liu, and T.K. Zimmermann. Lagrangian-eulerian finite element formulations for incompressible viscous flows. Computer Methods in Applied Mechanics and Engineering, 29:329-349, 1981.

[Hro] J. Hron. private communication. 2010.

[HRT92] J.G. Heywood, R. Rannacher, and S. Turek. Artificial boundaries and flux and pressure conditions for the incompressible Navier-Stokes equations. Int. J. Numer. Math. Fluids, 22:325-352, 1992.

[HT06] J. Hron and S. Turek. A monolithic fem/multigrid solver for an ale formulation of fluid-structure interaction with applications in biomechanics. In H.-J. Bungartz and M. Schäfer, editors, Fluid-Structure Interaction: Modeling, Simulation, Optimization, Lecture Notes in Computational Science and Engineering, pages 146-170. Springer, 2006.

$\left[\mathrm{HTM}^{+} 10\right]$ J. Hron, S. Turek, M. Madlik, M. Razzaq, H. Wobker, and J.F. Acker. Numerical simulation and benchmarking of a monolithic multigrid solver for fluid-structure interaction problems with application to hemodynamics. In H.-J. Bungartz and M. Schäfer, editors, Fluid-Structure Interaction II: Modeling, Simulation, Optimization, Lecture Notes in Computational Science and Engineering, pages 197-220. Springer, 2010.

[KR10] M. Kimmritz and T. Richter. Parallel multigrid method for finite element simulations of complex flow problems on locally refined meshes. Numerical Linear Algebra with Applications, 2010. doi: 10.1002/nla.744.

[OWA93] J.T. Oden, W. Wu, and M. Ainsworth. An a posteriori error estimate for finite element approximations of the navier-stokes equations. Computer Methods in Applied Mechanics and Engineering, 111:185-202, 1993.

[Ric11] T. Richter. A monolithic multigrid solver for 3d fluid-structure interaction problems. submitted to Siam J. Scientific Computing, 2011. 
[RS97] R. Rannacher and F.-T. Suttmeier. A feed-back approach to error control in finite element methods: Application to linear elasticity. Computer Methods in Applied Mechanics and Engineering, 19:434-446, 1997.

[RW10] T. Richter and T. Wick. Finite elements fo fluid-structure interaction in ale and fully eulerian coordinates. Computer Methods in Applied Mechanics and Engineering, 2010. doi: 10.1016/j.cma.2010.04.016).

$\left[\mathrm{THR}^{+} 10\right]$ S. Turek, J. Hron, M. Razzaq, H. Wobker, and M. Sch" afer. Numerical benchmarking of fluid-structure interaction: A comparison of different discretization and solution approaches. In H.J. Bungartz, M. Mehl, and M. Schäfer, editors, Fluid Structure Interaction II: Modeling, Simulation and Optimization. Springer, 2010.

[Ver96] R. Verfürt. A Review of A Posteriori Error Estimation and Adaptive MeshRefinement Techniques. Wiley/Teubner, New York-Stuttgart, 1996.

[Wal99] W.A. Wall. Fluid-Structure Interaction with Stabilized Finite Elements. PhD thesis, University of Stuttgart, 1999. urn:nbn:de:bsz:93-opus-6234.

[Wic11] T. Wick. Fluid-structure interactions using different mesh motion techniques. Computers and Structures, 89:1456-1467, 2011. doi: 10.1016/j.compstruc.2011.02.019. 